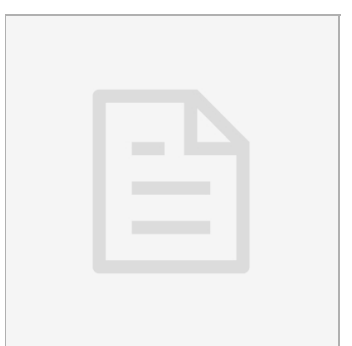

VERSION 1

AUG 20, 2019

open $\boldsymbol{\text { ACCESS }}$

DOI:

dx.doi.org/10.17504/protoco s.io.smdec 26

Protocol Citation: Xinyu Zhang, Ying Hu, Ke Xu 2019. DNA Methylation Signatures Predict HIV Prognosis and Mortality. protocols.io https://dx.doi.org/10.17504/p rotocols.io.smdec26

License: This is an open access protocol distributed under the terms of the Creative Commons Attribution License, which permits unrestricted use, distribution, and reproduction in any medium, provided the original author and source are credited

Protocol status: Working We use this protocol and it's working

Created: Aug 14, 2018

Last Modified: Aug 20 2019

PROTOCOL integer ID: 14725

\section{(3) DNA Methylation Signatures Predict HIV Prognosis and Mortality V.1}

$\rightarrow$ In 1 collection

$$
\mathrm{Ke}
$$

Xinyu Zhang ${ }^{1}$, Ying $\mathrm{Hu}^{2}, \mathrm{Xu}^{1}$

${ }^{1}$ Yale University; ${ }^{2}$ National Cancer Institute

Metabolomics Protocols \& Workflows

Tech. support email: bbmisraccb@gmail.com

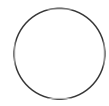

Xinyu Zhang

\section{ABSTRACT}

Background The effects of tobacco smoking upon epigenome-wide methylation signatures in white blood cells (WBCs) collected from persons living with HIV may have important implications for their immune-related outcomes, including frailty and mortality. The application of a machine learning approach to the analysis of CpG methylation in the epigenome enables the selection of phenotypically relevant features from high-dimensional data. Using this approach, we now report that a set of smoking-associated DNA-methylated CpGs predicts HIV prognosis and mortality in an HIV-positive veteran population.

Methods and Findings A total of 1,137 DNA samples were extracted from WBCs in HIV-positive male veterans. Here, HIV prognosis was measured by Veteran Aging Cohort Study Index that was highly correlated with HIV disease frailty. We first conducted two epigenome-wide association studies (EWAS) for tobacco smoking in two HIV-positive sample sets. We then selected smokingassociated DNA methylation features using a machine learning approach to differentiate good and poor HIV prognosis and to predict mortality. The performance of the prediction was validated in an independent sample set. We identified137epigenome-widesignificant CpGs in meta-EWAS analysis for smoking in HIV-positive samples ( $<<1.70 E-07)$. Using an in-house developed bioinformatic pipeline, we selected $698 \mathrm{CpG}$ sites that predicted high HIV frailty [(Area Under Curve (AUC) = 0.73, (95\%Cl: 0.63 0.83)] and was replicated in an independent sample [(AUC $=0.78,95 \% \mathrm{Cl}: 0.73 \sim 0.83)]$. We further found an association of a DNA methylation index constructed from the 698-CpGs that were associated with a 5-year survival rate [HR $=1.46 ; 95 \% \mathrm{Cl}: 1.06 \sim 2.02, \mathrm{p}=0.02]$. Interestingly, the 698CpGs located on 445 genes were enriched on the integrin signaling pathway $(p=9.55 E-05$, False discovery rate=0.036), which is responsible for regulation of the cell cycle, differentiation, and adhesion.

ConclusionWe demonstrated that smoking-associated DNA methylation features in white blood cells predict HIV infection prognosis and mortality in a population living with HIV. Feature selection is a powerful approach to identify DNA methylation biomarkers for the prediction of medical outcomes.

\title{
Workflow
}




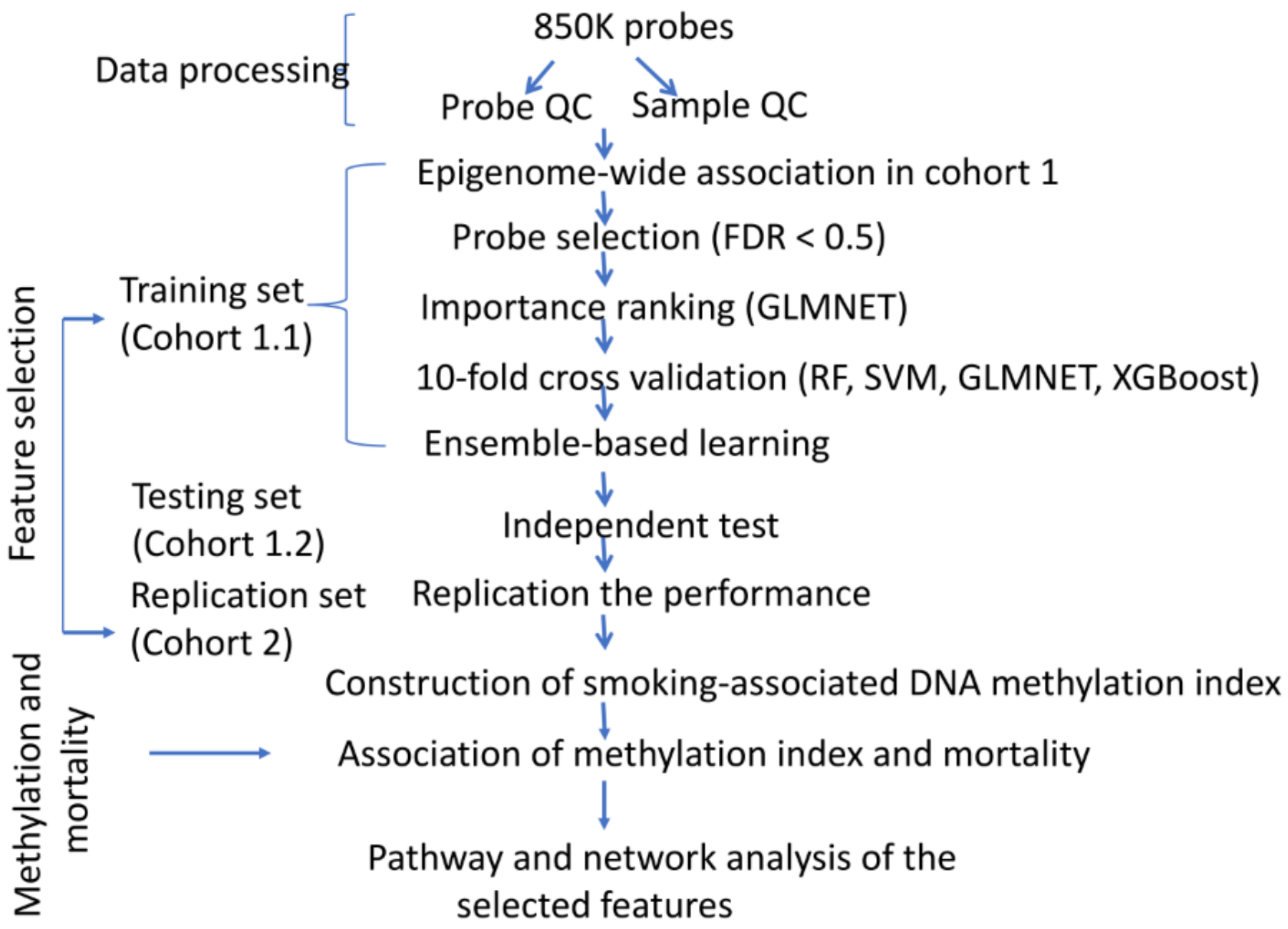

\section{Epigenome-wide Association Analysis}

2 Following protocols of https://www.nature.com/protocolexchange/protocols/6335

\section{Meta-analysis}

3 We conducted an EWAS meta-analysis by combining the data from the discovery and replication samples. Effect size and p-values for each probe were obtained from analyses in cohort 1 and cohort 2 samples, respectively. We performed fixed-effects, inverse-variance meta-analysis, with scheme parameters of sample size and standard error by implementing the METAL (ver: 2010-02-08) program, combining summary statistics in two sample sets. We investigated heterogeneity in two sample sets using the I2-statistic.

Machine Learning Selection of Smoking-association DNA Methylation for HIV Prognosis us.

We developed an in-house ensemble-based feature selection R package, SmartFeatureSelction

(https://bitbucket.org/starrcofly/smartfeatureselection.git)to link smoking-associated CpG sites to HIV outcomes. SmartFeatureSelectionused a supervised machine learning approach and merges EWAS with step-wise feature selection. The protocol included: 1) filtering of irrelevant probes based on methylation association analysis; 2) consideration of the relative attribution of each probe to rank the importance of the probes; 3 ) application of multiple machine learning methods to optimize the model; 4) building a model based on greedy ensemble by applying a weighted average of 4 machine learning models; 5) validation of the model in two independent samples. 
Considering the samples were processed at different times and platforms, batch effects were removed using Function removeBatchEffect in $\mathrm{R}$ limma (ver. 3.32.10) library before performing machine learning prediction. To reduce redundant DNA methylation signals and noise for improving the prediction accuracy of HIV frailty, CpG sites with FDR $<0.5$ from EWAS in cohort 1 were selected for machine learning. The samples in cohort 1 were randomly divided into a training set and a test setwith ratio of 8:2. We first built a model using the training set, in which each sample was labeled poor (VACS index $>50$ ) or good prognosis (VACS index $<=50$ ). We then tested the model by performing 10 -fold cross validation in the testing set, and the best-performed model was tested in an independent replication set.

\section{Association of Smoking-associated DNA Methylation Index and Mortality}

5 To examine whether the select CpG site methylation was associated with mortality, we constructed a methylation index from the selected smoking-associated $\mathrm{CpG}$ sitesfollowing the previous formula[34]. A separate index was constructed for hypomethylated and hypermethylated $\mathrm{CpG}$ sites, respectively.

The association of the DNA methylation risk index with all-cause mortality was examined by Kaplan-Meier plots and log-rank tests in all samples. Cox regression model was then used to adjust for age, antiretroviral therapy, HIV-1 loads, and CD4 counts. In the Cox regression model, the DNA methylation index score was a categorical variable (using the highest quartiles as the reference category) or a continuous variable (calculating HR

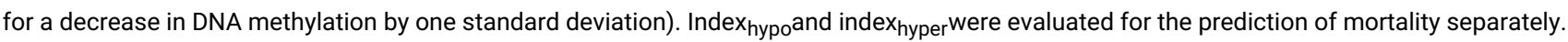

\section{Gene Enrichment Analysis}

6 Pathway and network analysis was conducted for the selected CpG sites on the nearest genes by employing Ingenuity Pathway Analysis (IPA). For genes with multiple $\mathrm{CpG}$ sites, the lowest p-value at the $\mathrm{CpG}$ site within a gene was used to represent the gene level significance. Significant pathways were defined at a FDR $<0.05$. 